\title{
ЛОКАЛІЗАЦІЯ СФЕРИЧНОЇ ФЕРОМАГНІТНОЇ МІКРОЧАСТИНКИ ПІД ДІЕЮ ШВИДКО ОСЦИЛЮЮЧОГО МАГНІТНОГО ПОЛЯ В ПОТОЦІ РІДИНИ
}

\author{
О.Ю. ГОРОБЕЦЬ, М.М. ПОТЬОМКІН
}

Запропоновано модель магнітного пінцета в зовнішньому швидко осцилюючому магнітному полі, яка дозволяє досліджувати рух магнітом'якої феромагнітної мікрочастинки в магнітній пасці та локалізувати її в умовах потоку рідини. Зроблено оцінки сили та частоти зовнішнього швидко осцилюючого магнітного поля, а також розміри сферичної мікрочастинки. Розраховано траєкторію руху сферичної мікрочастинки.

\section{1. Вступ}

Одним з перспективних методів досліджень, що знаходить застосування в різних галузях науки [1-3], є локалізація мікрочастинок за допомогою магнітної пастки. Його особливістю, порівняно з іншими методами, є можливість маніпулювання окремими мікрочастинками, що дозволяе досліджувати фізичні та біологічні властивості клітини в природному середовищі, вивчати механічні та адсорбційні властивості ДНК та інше [4].

Однак, з метою забезпечення умов, сприятливих для живих клітин, такі дослідження часто здійснюють у проточній рідині [5-7], що вимагає враховувати під час використання й проектування магнітних пасток додаткової сили, зумовленої наявним гідравлічним потоком. Тому розробка нових і вдосконалення існуючих методик дослідження магнітних пасток з урахуванням впливу сукупності сил, які діють на мікрочастинку, є актуальним науковим завданням.

Для того щоб локалізувати в просторі мікрочастинку, яка перебуває під дією магнітного поля, повинна виконуватися умова існування точки, в якій потенці- альна енергія досягає свого локального мінімуму, тобто утворюється потенціальна яма. Оскільки в постійному магнітному полі для феро- і парамагнітних мікрочастинок досягти такого локального мінімуму неможливо [8], у даній роботі пропонується використовувати змінне магнітне поле аналогічно методу, що запропонував Капиця [9] для стабілізації верхнього нестійкого положення математичного маятника. Як буде показано далі, це надає можливість, створивши ефективний локальний мінімум енергії, вирішити питання локалізації сферичної мікрочастинки за допомогою змінного осцилюючого магнітного поля.

\section{2. Розрахункова частина}

Для дослідження можливості практичної реалізації магнітного поля з локальним мінімумом енергії, у першу чергу, розглянемо феромагнітну мікрочастинку в магнітному полі, що залежить від просторової координати $\mathbf{r}$. Тоді енергія $U$ цієї мікрочастинки буде мати вигляд [10]:

$U(\mathbf{r})=-\mathbf{M H}$,

де $\mathbf{H}$ - напруженість магнітного поля, $\mathbf{M}$ - магнітний момент мікрочастинки.

Вважатимемо, що якщо стала складова $z$ компоненти магнітного поля більша по відношенню до $x$ i $y$ компонент, то можна вважати мікрочастинку намагніченою вздовж осі $z$ однорідно і її розміри набагато менші, ніж характерний масштаб зміни магнітного поля. Магнітний момент мікрочастинки $M_{0}$ фіксований вздовж осі $O z$ за рахунок підмагнічування. Тоді 
в неоднорідному осцилюючому магнітному полі виникає осцилююча сила:

$\mathbf{f}(\mathbf{r}, t)=-\frac{d}{d \mathbf{r}}\left(M_{0} H_{z}(x, y, z, t)\right)$,

де $z$-компонента зовнішнього магнітного поля матиме вигляд $H_{z}=h_{z}(x, y, z) \cos (\omega t)$.

3 огляду на це, рівняння руху буде мати загальновідомий вигляд

$m \ddot{\mathbf{r}}+\alpha\left(\dot{\mathbf{r}}-\mathbf{V}_{0}\right)=\mathbf{f}(\mathbf{r}, t)$,

де $m$ - маса мікрочастинки, $\alpha$ - дисипативний множник, $\dot{\mathbf{r}}$ - перша похідна за часом від просторової координати, $\ddot{\mathbf{r}}$ - друга похідна за часом від просторової координати, $\mathbf{V}_{0}$ - швидкість потоку рідини. 3 урахуванням введених вище позначень маємо

$\mathbf{f}(\mathbf{r}, t)=-\frac{d U}{d \mathbf{r}}=M_{0} \frac{d}{d \mathbf{r}} h_{z}(x, y, z) \cos (\omega t)$,

де $\frac{d}{d \mathrm{r}} h_{z}(x, y, z)$ - деяка функція координат.

Аналіз руху мікрочастинки може бути значно спрощений, аналогічно задачі Капиці [9]. Зважаючи на те, що рух мікрочастинки являе собою переміщення уздовж плавної траєкторії з одночасними малими осциляціями (із частотою $\omega$ ) навколо неї, функцію $\mathbf{r}$ представимо у вигляді суми:

$\mathbf{r}=\mathbf{R}+\boldsymbol{\xi}$

де $\mathbf{R}$ - функція, яка описує "плавний" рух мікрочастинки, $\boldsymbol{\xi}$ - швидко осцилююча компонента, $|\xi| \ll|\mathbf{R}|$.

Підставимо (3) в (1) i, розклавши $\frac{d}{d \mathbf{r}} U(\mathbf{r})$ та $\mathbf{f}(\mathbf{r}, t)$ по малих зміщеннях $\xi$, з точністю до квадратичних по $\xi$ членів отримаємо

$m \ddot{\mathbf{R}}+\alpha\left(\dot{\mathbf{R}}-\mathbf{V}_{0}\right)+m \ddot{\boldsymbol{\xi}}+\alpha \dot{\boldsymbol{\xi}}=\mathbf{f}(\mathbf{R}, t)+\frac{\partial}{\partial x_{i}} \boldsymbol{f}(\mathbf{R}, t) \boldsymbol{\xi}_{i}$,

де $x_{i}$ - компоненти просторового вектора $\mathbf{r}, i=1,2,3$.

Виділивши з отриманого виразу (4) осцилюючі члени, отримаємо

$m \ddot{\boldsymbol{\xi}}+\alpha \dot{\boldsymbol{\xi}}=\mathbf{f}(\mathbf{R}, t)$.

Розв'язок цього диференціального рівняння будемо шукати у вигляді

$\boldsymbol{\xi}=\mathbf{A} \cos \omega t+\mathbf{B} \sin \omega t$.

Взявши похідні від виразу (6) і підставивши їх у вираз (5), отримаємо систему

$$
\left\{\begin{aligned}
-\mathbf{A} m \omega^{2}+\mathbf{B} \alpha \omega & =M_{0} \frac{d}{d \mathbf{r}} h_{z}(x, y, z), \\
-\mathbf{B} m \omega^{2}-\mathbf{A} \alpha \omega & =0 .
\end{aligned}\right.
$$

Розв'язавши її, отримаємо значення коефіцієнтів А і B:

$\mathbf{A}=-\frac{m M_{0} \frac{d}{d \mathbf{r}} h_{z}(x, y, z)}{m^{2} \omega^{2}+\alpha^{2}}, \quad \mathbf{B}=\frac{\alpha M_{0} \frac{d}{d \mathbf{r}} h_{z}(x, y, z)}{m^{2} \omega^{3}+\alpha^{2} \omega}$.

Отримані коефіцієнти А і В підставимо у вираз (6) і отримаємо вираз для осцилюючої компоненти:

$\boldsymbol{\xi}(t)=-\frac{m M_{0} \frac{d}{d \mathbf{r}} h_{z}(x, y, z)}{m^{2} \omega^{2}+\alpha^{2}} \times$

$\times \cos \omega t+\frac{\alpha M_{0} \frac{d}{d \mathbf{r}} h_{z}(x, y, z)}{m^{2} \omega^{3}+\alpha^{2} \omega} \sin \omega t$.

Позначимо $|\sigma|=\frac{m M_{0}^{2}}{4\left(m^{2} \omega^{2}+\alpha^{2}\right)}$, тоді $\mathbf{A}$ і $\mathbf{B}$ перепишуться і такому вигляді:

$\mathbf{A}=\frac{4|\sigma| \frac{d}{d \mathbf{r}} h_{z}(x, y, z)}{M_{0}}, \quad \mathbf{B}=\frac{4 \alpha|\sigma| \frac{d}{d \mathbf{r}} h_{z}(x, y, z)}{m M_{0} \omega}$

Перепишемо вираз (6) у такому вигляді:

$\boldsymbol{\xi}=\left(A_{0} \cos \omega t+B_{0} \sin \omega t\right) \frac{d}{d \mathbf{r}} h_{z}(x, y, z)$,

де $A_{0}=\frac{4|\sigma|}{M_{0}}, B_{0}=\frac{4|\sigma|}{M_{0}} \frac{\alpha}{m \omega}$.

Тоді з (9) маємо

$\boldsymbol{\xi}=\sqrt{A_{0}^{2}+B_{0}^{2}}\left(\frac{A_{0}}{\sqrt{A_{0}^{2}+B_{0}^{2}}} \cos \omega t+\right.$

$\left.+\frac{B_{0}}{\sqrt{A_{0}^{2}+B_{0}^{2}}} \sin \omega t\right) \frac{d}{d \mathbf{r}} h_{z}(x, y, z)$,

де $\cos \alpha_{0}=\frac{A_{0}}{\sqrt{A_{0}^{2}+B_{0}^{2}}}=\frac{m \omega}{\sqrt{m^{2} \omega^{2}+\alpha^{2}}}$.

Згорнувши попередній вираз тригонометричними перетвореннями, отримаємо

$\boldsymbol{\xi}=\sqrt{A_{0}^{2}+B_{0}^{2}} \cos \left(\omega t-\alpha_{0}\right) \frac{d}{d \mathbf{r}} h_{z}(x, y, z)$,

де $\alpha_{0}-$ зсув фази. Підставивши в (10) коефіцієнти $A_{0}=\frac{4|\sigma|}{M_{0}}, B_{0}=\frac{4|\sigma|}{M_{0}} \frac{\alpha}{m \omega}$ та $|\sigma|=\frac{m M_{0}^{2}}{4\left(m^{2} \omega^{2}+\alpha^{2}\right)}$ остаточний вираз для $\boldsymbol{\xi}$ набуде такого вигляду:

$\boldsymbol{\xi}=\frac{M_{0}}{\omega \sqrt{m^{2} \omega^{2}+\alpha^{2}}} \cos \left(\omega t-\alpha_{0}\right) \frac{d}{d \mathbf{r}} h_{z}(x, y, z)$

або

$\boldsymbol{\xi}=\frac{4 \delta}{M_{0}} \cos \left(\omega t-\alpha_{0}\right) \frac{d}{d \mathbf{r}} h_{z}(x, y, z)$,

де $\delta=\frac{M_{0}^{2}}{4 \omega \sqrt{m^{2} \omega^{2}+\alpha^{2}}}$.

ISSN 2071-0194. Укр. фіз. журн. 2012. T. 57, №9 
Підставимо (11) в рівняння руху (4) і проведемо усереднення, маємо

$\frac{\partial}{\partial x_{i}} \mathbf{f}(\mathbf{R}, t) \boldsymbol{\xi}_{i}=2 \delta \frac{d}{d \mathbf{r}}\left[\frac{d}{d \mathbf{r}} h_{z}(x, y, z)\right]^{2} \times$

$\times\left[\overline{\cos ^{2}(\omega t)} \cos \alpha_{0}-\overline{\cos (\omega t) \sin (\omega t)} \sin \alpha_{0}\right]$,

звідки

$\frac{\partial}{\partial x_{i}} \mathbf{f}(\mathbf{R}, t) \boldsymbol{\xi}_{i}=\delta \frac{d}{d \mathbf{R}}\left[\frac{d}{d \mathbf{R}} h_{z}(x, y, z)\right]^{2} \times$

$\times \cos \alpha_{0}=\delta_{0} \frac{d}{d \mathbf{R}}\left[\frac{d}{d \mathbf{R}} h_{z}(x, y, z)\right]^{2}$,

де $\delta_{0}=\frac{m M_{0}^{2}}{4\left(m^{2} \omega^{2}+\alpha^{2}\right)}$.

Тоді рівняння для усередненого руху буде мати вигляд

$m \ddot{\mathbf{R}}+\alpha(\dot{\mathbf{R}}-\mathbf{V})=-\delta_{0} \frac{d}{d \mathbf{R}}\left(\frac{d h_{z}}{d \mathbf{R}}\right)^{2}$.

Візьмемо координатну залежність $h_{z}(x, y, z)=$ $a\left(-z^{2}+\frac{x^{2}+y^{2}}{3}\right)$, тоді стаціонарна точка $Z_{0}$ записується у вигляді

$\alpha V_{0 z}=8 \delta_{0} a^{2} Z_{0}$

Звідки маємо $Z_{0}$ :

$Z_{0}=\frac{\alpha V_{0 z}}{8 \delta_{0} a^{2}}$

Аналогічно для стаціонарних точок

$X_{0}, Y_{0}: \alpha V_{0 x}=\frac{8}{9} \delta_{0} a^{2} X_{0}$.

Звідки $X_{0}, Y_{0}$ :

$X_{0}=Y_{0}=\frac{9}{8} \frac{\alpha V_{0 x}}{\delta_{0} a^{2}}$.

Підставивши $\delta_{0}$, отримаємо остаточний вигляд стаціонарних точок:

$X_{0}=Y_{0}=\frac{9}{2} \frac{\alpha V_{0 x}\left(m^{2} \omega^{2}+\alpha^{2}\right)}{a^{2} M_{0}^{2} m}$,

$\mathrm{Z}_{0}=\frac{1}{2} \frac{\alpha V_{0 x}\left(m^{2} \omega^{2}+\alpha^{2}\right)}{a^{2} M_{0}^{2} m}$.

Розглянемо амплітуди осциляцій для стаціонарних точок. Вираз для амплітуд малих осциляцій навколо стаціонарної точки $X \approx X_{0}$ випишемо із виразу (11) для $\xi$ :

$\xi_{x}=\xi_{y}=\frac{4 \delta}{M_{0}} \frac{d}{d x} h_{z}(x, y, z)=\frac{3 \alpha V_{0 x} \sqrt{m^{2} \omega^{2}+\alpha^{2}}}{m \omega a M_{0}}$, $\xi_{z}=\frac{4 \delta}{M_{0}} \frac{d}{d z} h_{z}(x, y, z)=\frac{3 \alpha V_{0 x} \sqrt{m^{2} \omega^{2}+\alpha^{2}}}{m \omega a M_{0}}$.

Із (12) знайдемо частоти малих коливань навколо стаціонарної точки по осі $O x$, маємо

$m \ddot{x}_{1}+\alpha\left(\dot{x}_{1}-V_{0 x}\right)=-\frac{8}{9} a^{2} \delta_{0} X_{0}-\frac{8}{9} a^{2} \delta_{0} x_{1}$.

Звідки

$\ddot{x}_{1}+\frac{\alpha}{m} \dot{x}_{1}+\frac{8}{9} \frac{a^{2} \delta_{0}}{m} x_{1}=0$.

Частота малих коливань:

$\Omega_{x}=\sqrt{\frac{8}{9} \frac{a^{2} \delta_{0}}{m}-\left(\frac{\alpha}{2 m}\right)^{2}}$,

при $\frac{\alpha}{2} \ll \frac{8 a^{2} \delta_{0}}{9}$, тому $\Omega_{x} \approx \Omega_{y} \approx \sqrt{\frac{8}{9} \frac{a^{2} \delta_{0}}{m}} \approx \sqrt{\frac{2}{9}} \frac{a M_{0}}{m \omega}$, оскільки $\lim _{\alpha \rightarrow 0} \delta_{0} \rightarrow \frac{M_{0}^{2}}{4 \omega^{2} m}$.

Оскільки $\frac{\alpha}{2} \ll 8 a^{2} \delta_{0}$, тому $\Omega_{z} \approx \sqrt{\frac{8 a^{2} \delta_{0}}{m}} \approx \sqrt{2} \frac{a M_{0}}{m \omega}$.

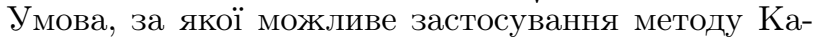
пиці являє собою умову, при якій частота повільних малих коливань набагато менша, ніж частота швидких коливань для $\boldsymbol{\xi}$, тобто $\Omega \ll \omega$. Тому

$\sqrt{\frac{2}{9}} \frac{a M_{0}}{m \omega} \ll \omega$

звідки маємо $\omega \gg \sqrt{\frac{a M_{0}}{m}}$.

\section{3. Чисельне моделювання магнітної пастки}

В основу моделі "магнітної пастки" було покладено рівняння руху мікрочастинки в магнітному полі (1). Проведемо точне чисельне моделювання "магнітної пастки" з осцилюючою силою (3), тоді рівняння руху мікрочастинки в магнітному полі матиме вигляд

$m \ddot{\mathbf{r}}+\alpha\left(\dot{\mathbf{r}}-\mathbf{V}_{0}\right)=M_{0} \frac{d}{d \mathbf{r}} h_{z}(x, y, z) \cos (\omega t)$.

Розглянемо випадок зі сферичною мікрочастинкою. Пронормуємо вираз (13), для цього введемо такі заміни: $\mathbf{r}=\beta \boldsymbol{\rho}$ (де $\boldsymbol{\rho}$ - радіус-вектор), $\omega t=\tau$ і коефіцієнт опору середовища запишемо через силу Стокса [11], що діє на сферичну мікрочастинку, яка рухається відносно рідини $\alpha=6 \pi \eta b$ ( $\eta$ - коефіцієнт динамічної в'язкості рідини) [11]. Отримаємо таке пронормоване рівняння руху мікрочастинки в безрозмірних величинах:

$\ddot{\boldsymbol{\rho}}+K\left(\dot{\boldsymbol{\rho}}-v_{0}\right)=D \frac{d}{d \boldsymbol{\rho}} h_{z}\left(\rho_{x}, \rho_{y}, \rho_{z}\right) \cos (\tau)$, 


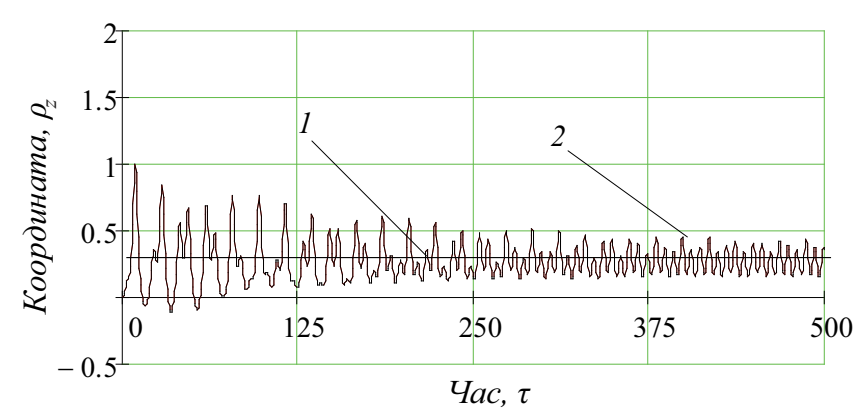

Рис. 1. Траєкторія руху мікрочастинки вздовж осі $z$ у кровотоку: 1 - стаціонарна точка траєкторіі, побудована за аналітичним виразом (13), 2 - траєкторія, побудована на результаті чисельного розрахунку рівняння (14). (Координати вказані в безрозмірних величинах)

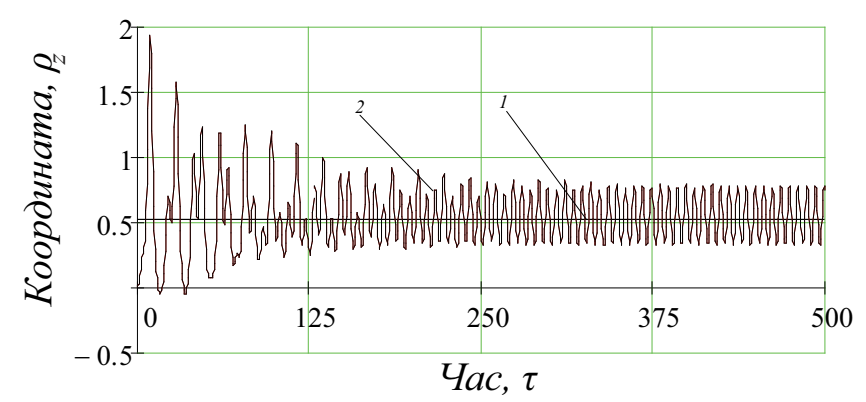

Рис. 2. Траєкторія руху мікрочастинки вздовж осі $z$ у водяному потоці: 1 - стаціонарна точка траєкторії, побудована за аналітичним виразом (13), 2 - траєкторія, побудована на результаті чисельного розрахунку рівняння (14). (Координати вказані в безрозмірних величинах)

де $K=\frac{\alpha}{m \omega}$ i $D=\frac{2}{9} \frac{M_{0} a}{m \omega^{2}}$ керуючі безрозмірні параметри, від яких залежить динаміка руху мікрочастинки.

Будемо вважати, що вектор швидкості $\mathbf{V}=\dot{\mathbf{r}}$ поступального руху мікрочастинки в кожний момент часу $\tau$, спрямований вздовж сили, визначається виразом у правій частині рівняння (15). Дослідження поведінки сферичної феромагнітної мікрочастинки в магнітній пасці проводилися з використанням програмного пакета MathCad 14.

Для наглядного прикладу, розглянемо поведінку "магнітної пастки" беручи до уваги умови, які властиві кровоносній судині людини. Прикладом буде слугувати вена. Діаметр вени 0,5 см, максимальна швидкість потоку крові в ній до 20-050 см/с [12]. Параметри системи: $b=0,05 \mathrm{~cm}, \rho_{\mathrm{Fe}}=7,87 \mathrm{\Gamma} / \mathrm{cm}^{3}$, $\mu_{\mathrm{Fe}}=1600 \Gamma \mathrm{c}, \omega=100$ Гц, $a=10 \Gamma / \mathrm{cm}^{5} \mathrm{c}^{2}, z_{0}=0$, $V_{z 0}=2 \mathrm{~cm} / \mathrm{c}, \beta=2$. Динамічна в'язкість крові становить $\eta=5 \cdot 10^{-3}$ г/см.с. Радіус мікрочастинки становить $10 \%$ від діаметра вени. Траєкторію руху сфери-

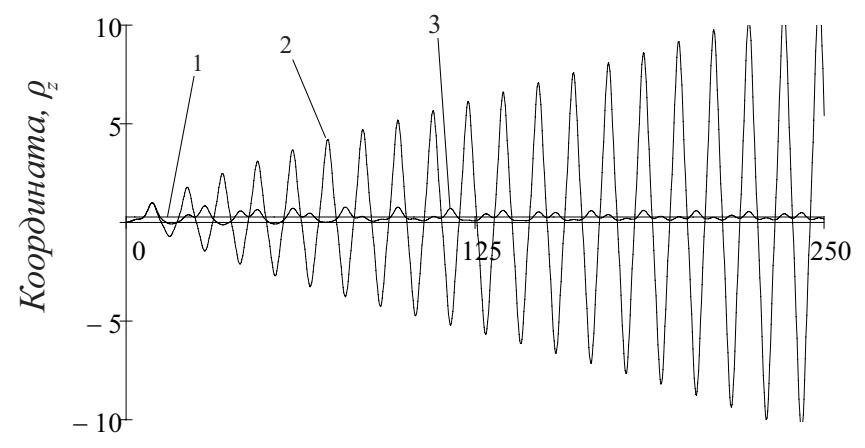

Yac, $\tau$

Рис. 3. Траєкторія руху мікрочастинки вздовж осі $z$ у кровотоку: 1 - стаціонарна точка траєкторії, побудована за аналітичним виразом (13), 2 - траєкторія побудована на результаті чисельного розрахунку рівняння (15) при невиконанні умови (14), 3 - траєкторія, побудована при виконанні умови (14) на результаті чисельного розрахунку рівняння (15). (Координати вказані в безрозмірних величинах)

чної мікрочастинки з заліза, за умови, що $H_{0} \gg 3$ Гс, наведено на рис. 1 та 2.

Аналізуючи траєкторії руху мікрочастинки 3 наведених вище рисунків, видно, що в умовах наявності кровотоку (рис. 1) локалізація феромагнітної мікрочастинки відбувається за 5 с, максимальна амплітуда коливань навколо стаціонарної точки в координаті 0,28 см становить 0,88 см і 0,28 см при стабільному коливальному режимі. Вихід на стабілізуючий режим відбувається за 3 сек. При зміні коефіцієнта в'язкості крові на в'язкість води $\eta=10^{-2} \Gamma / \mathrm{cm} \cdot \mathrm{c}$ (рис. 2) стаціонарна точка змінила своє положення на координату 0,55 і при цьому максимальна амплітуда коливань навколо стаціонарної точки збільшилася і становить $1,88 \mathrm{~cm}$ та $0,49 \mathrm{~cm}$ при стабільному коливальному режимі. Вихід на стабілізуючий режим відбувається за 2,5 с.

Наведені вище рисунки зображують траєкторії руху мікрочастинки в потоці рідини за виконання умови (14). Покажемо як поводить себе мікрочастинка, коли умова (14) не виконується (рис. 3).

На рис. 3 видно, що при виконанні умови (14) можлива локалізація мікрочастинки (коливальний процес зображений траєкторією 3) в області стаціонарної точки $Z_{0}$ (пряма 1 ), при цьому амплітуда коливань не виходить за межі вузького діапазону в області $Z_{0}$. Крім того, коливання відбувається в стабільному режимі з явним затуханням після 4 с, що свідчить про захоплення мікрочастинки в "магнітну пастку". У випадку невиконання умови (14) вплив "магнітної пастки" на мікрочастинку спостерігається, але її захо- 
плення не відбувається, при цьому амплітуда коливання відразу виходить за межі допустимого діапазону.

Аналіз графіків показує, що в вибраному діапазоні початкових даних система дійсно демонструє властивості магнітної пастки шляхом утримання магнітної мікрочастинки в локалізованій області магнітного поля. Видно, що час виходу системи у стабільний локалізований режим змінюється залежно від початкових умов, які залежать від таких параметрів системи, як радіус мікрочастинки, динамічна в'язкість рідини, густини матеріалу, частоти зовнішнього магнітного поля та ін. При цьому незначна зміна одного з параметрів приводить до значної зміни, наприклад, значень амплітуд і координати, при якій відбувається подальше коливання мікрочастинки.

3 рисунків видно, що накладення магнітного поля на мікрочастинку приводить до локалізації точки їі знаходження, при цьому мікрочастинка здійснює осцилюючі коливання в невеликому діапазоні координат. Діапазон радіуса сферичної мікрочастинки, для можливості її локалізації, становить $3-10^{-2}$ $6 \cdot 10^{-2}$ см (6-12\% від діаметра вени), при цьому локалізація мікрочастинки можлива в межах $0<z<$ 30 см і $0<x<0,5$ см. Час локалізації від 2 с до 5 с, якщо величина поля $H_{0} \gg 6$ Гс при радіусі мікрочастинки $3 \cdot 10^{-2}$ і $H_{0} \gg 2$ Гс при радіусі мікрочастинки $6 \cdot 10^{-2}$ cм.

\section{4. Висновки}

Отримані результати досліджень динаміки руху сферичної феромагнітної мікрочастинки як теоретично, так і чисельно показують, що використовуючи метод швидко осцилюючого поля [9], можна досягти локального мінімуму потенціальної енергї, що дозволяє здійснювати локалізацію в заданій точці простору магнітною пасткою сферичної феромагнітної мікрочастинки для широкого діапазону параметрів (таких як радіус мікрочастинки, щільність і намагнічування насичення матеріалу, з якого вона зроблена, частота осциляції магнітного поля, швидкість та в'язкість крові в кровоносній судині). Локалізацію феромагнітних мікрочастинок можливо досягти, при частоті й величині поля $\omega=100$ Гц і $H_{0} \gg 2$ Гс відповідно, для судин, діаметр яких близький до діаметра вени.

1. Б.А. Каррерас, Д. Ньюман, В.Е. Линч, П.Х. Даймонд, Физика плазмы 22, 819 (1996).

2. Б.Б. Кадомцев, М.Б. Кадомцев, УФН 167, 649 (1996).

3. J. Zlatanova and S.H. Leuba, Biochem. Cell Biol. 81, 151 (2003).
4. C. Haber and D. Wirtz, Rev. Sci. Instrum 71, 4561 (2000).

5. C.S. Lee, H. Lee, and R.M. Westervelt, Applied physics letters 79, 3308 (2001).

6. H. Lee, A.M. Purdon, R.M. Westervelt, Applied physics letters 85, 1063 (2004).

7. T. Deng, G.M. Whitesides, Applied physics letters 78, 1775 (2001).

8. У.Д. Филипс, УФН 169, 305 (1999).

9. Л.Д. Ландау, Е.М. Лившиц, Механика (Наука, Москва, 1965).

10. А.И. Ахиезер, В.Г. Барьяхтар, С.В. Пелетминский, Спиновые волны (Наука, Москва, 1967).

11. Г. Ламб, Гидродинамика (ОГИЗ, Москва, 1947).

12. Н.Н. Савицкий, Биофизические основы кровообращения и клинические методы изучения гемодинамики (Медицина, Ленинград, 1963).

Одержано 09.04.11

ЛОКАЛИЗАЦИЯ СФЕРИЧЕСКОЙ ФЕРРОМАГНИТНОЙ МИКРОЧАСТИЦЫ ПОД ДЕЙСТВИЕМ БЫСТРО ОСЦИЛЛИРУЮЩЕГО МАГНИТНОГО ПОЛЯ В ПОТОКЕ ЖИДКОСТИ

О.Ю. Горобеи, М.М. Потемкин

$\mathrm{P}$ е з ю м е

Предложена модель магнитного пинцета во внешнем быстро осциллирующем магнитном поле, которая позволяет исследовать движение магнитомягкой ферромагнитной микрочастицы в магнитной ловушке и локализовать ее в условиях потока жидкости. Сделаны оценки силы и частоты внешнего быстро осциллирующего магнитного поля, а также размеры сферической микрочастицы. Рассчитана траектория движения сферической микрочастицы.

THE LOCALIZATION OF A SPHERICAL FERROMAGNETIC MICROPARTICLE IN AN OSCILLATING MAGNETIC FIELD IN A LIQUID STREAM

O.Yu. Gorobets, M.M. Potyemkin

National Technical University of Ukraine

"Kiev Polytechnic Institute"

(37, Peremogy av., Kyiv 0356, Ukraine;

e-mail: mike_potyemkin@mail.ru)

$\mathrm{S} \mathrm{u} \mathrm{m} \mathrm{m} \mathrm{a} \mathrm{r} \mathrm{y}$

The model of magnetic tweezers in a rapidly oscillating alternating magnetic field, which allows one to estimate the movement of a ferromagnetic magnetically soft microparticle in a magnetic trap and to localize it in a liquid, is offered. The estimations of the strength and the frequency of an oscillating alternating magnetic field and the size of a spherical microparticle are carried out. The movement trajectory of a spherical microparticle is calculated as well. 